\title{
INTRODUCTION \\ Imaging in neurosurgical disease
}

\author{
Joshua W. Osbun, MD, ${ }^{1}$ Ralph G. Dacey Jr., MD, ${ }^{2}$ Daniel L. Barrow, MD, ${ }^{3}$ \\ Amit M. Saindane, MD, MBA, ${ }^{4}$ and Christopher Nimsky, MD ${ }^{5}$
}

1Departments of Neurosurgery, Radiology, and Neurology, Washington University in St. Louis; ${ }^{2}$ Department of Neurosurgery, Washington University in St. Louis, Missouri; ${ }^{3}$ Department of Neurosurgery, Emory University; ${ }^{4}$ Department of Radiology and Imaging Sciences, Emory University, Atlanta, Georgia; and ${ }^{5}$ Department of Neurosurgery, University Marburg, Germany

$\mathrm{W}$ $\mathrm{E}$ as neurosurgeons tend to focus on technical and manual aspects of surgery, but a keen understanding of both basic and advanced imaging techniques is vital to a successful operation. In this issue of Neurosurgical Focus, we sought to solicit papers that highlighted how imaging enhances our practice as neurosurgeons-whether it be the preoperative workup of a cerebral aneurysm or brain tumor; intraoperative image guidance with navigation, ultrasound, functional tractography, and neuromonitoring; or angiography or postoperative imaging that allows for confirmation of lesion removal and/or disease prognostication. We intended to feature papers that reviewed tried-and-true standard imaging practices as well as those that emphasized avant-garde imaging practices. After final manuscript submission and review, we believe this issue serves as a comprehensive review of established imaging techniques for the planning, intraoperative guidance, and follow-up of intracranial surgery while highlighting cutting-edge imaging technologies, particularly in the realm of cerebrovascular and tumor neurosurgery. The call for papers generated a large number of submissions on the use of hybrid imaging techniques that combine nuclear medicine with traditional CT and MR modalities to evaluate patients for spinal fusion, for example, SPECT/CT and PET/MR. As the use of these techniques has begun to grow in spine surgery, we have showcased their emergence with both a review article and an original case series demonstrating their potential utility. While no single issue of this journal can encompass the entire breadth of neurosurgical imaging, we hope this issue gives the readers a sense of the recent advancements our field has made in utilizing radiographic imaging as a pillar of our practice.

https://thejns.org/doi/abs/10.3171/2019.9.FOCUS19764

\section{Disclosures}

The authors report no conflict of interest.

\section{Correspondence}

Joshua W. Osbun: josbun@wustl.edu. 